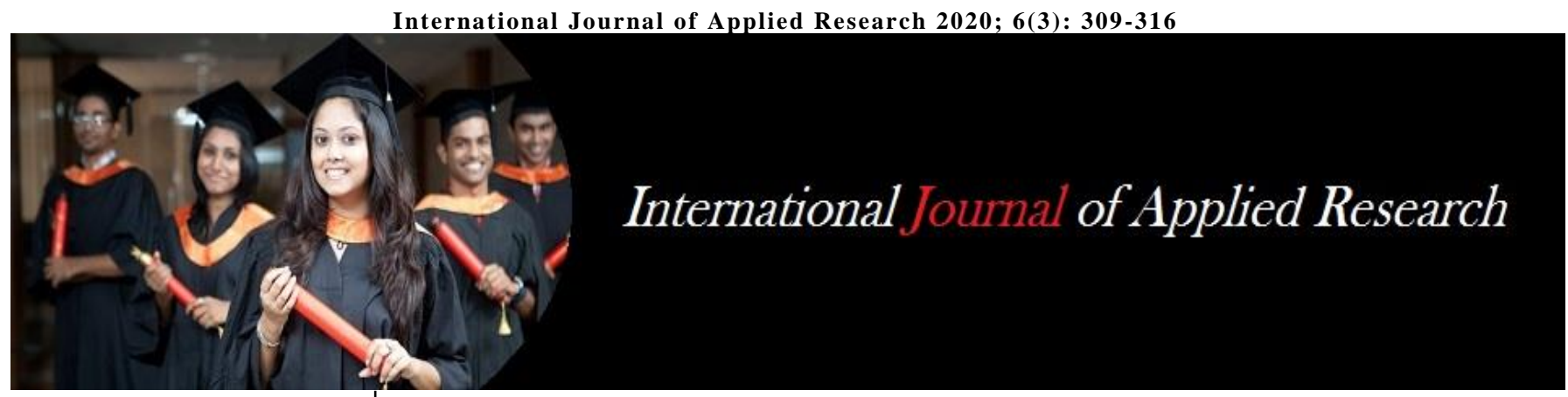

ISSN Print: 2394-7500 ISSN Online: 2394-5869 Impact Factor: 5.2

IJAR 2020; 6(3): 309-316 www.allresearchjournal.com

Received: 21-01-2020 Accepted: 25-02-2020

Orou Bienvenue Sabi YO Laboratoire d'Analyse et de Recherches sur les Dynamiques Economiques et Sociales (LARDES), Faculté d'Agronomie, Université de Parakou, BP : 123 Parakou, Bénin

Nasser Mohamed Baco Laboratoire Société-

Environnement (LaSEn) ; Faculté d'Agronomie ; Université de Parakou, BP : 123 Parakou, Bénin

Innocent Adédédji Labiyi Laboratoire d'Analyse et de Recherches sur les Dynamiques Economiques et Sociales (LARDES), Faculté d'Agronomie, Université de Parakou, BP : 123

Parakou, Bénin

Fabien C Hountondji

Faculté d'Agronomie, Université de Parakou, BP : 123 Parakou, Bénin

Jacob Afouda Yabi

Laboratoire d'Analyse et de

Recherches sur les Dynamiques

Economiques et Sociales

(LARDES), Faculté d'Agronomie,

Université de Parakou, BP : 123

Parakou, Bénin

Géry Van Nieuwenhuysen

Programme d'Appui au

Développement des Filières Agricoles au Bénin (PROFI)

Honorat Emongou

Programme d'Appui au

Développement des Filières

Agricoles au Bénin (PROFI).
Correspondence Author: Innocent Adédédji Labiyi Laboratoire d'Analyse et de Recherches sur les Dynamiques Economiques et Sociales (LARDES), Faculté d'Agronomie, Université de Parakou, BP : 123 Parakou, Bénin

\section{Effets environnementaux et production de nouvelles sources énergiques de la biomasse issue du riz : cas de l'étuvage du riz au CePEA Sourou au Nord-Ouest du Bénin}

\author{
Orou Bienvenue Sabi YO, Nasser Mohamed Baco, Innocent Adédédji \\ Labiyi, Fabien C Hountondji, Jacob Afouda Yabi, Géry Van \\ Nieuwenhuysen and Honorat Emongou
}

\begin{abstract}
Rice parboiling is a time and energy consuming activity, and is a source of solid and liquid wastes including husks, rice bran and wastewater. This research aims to assess the environmental effects of parboiling rice before and five years after the contractualization between rice producers and processors. The case study focused on the Center for the Promotion of Women's Agricultural Entrepreneurship (CePEA Sourou) in Natitingou in North-west Benin. The search for solutions to environmental effects, led CePEA to introduce innovations such as improved stoves, briquettes (bio-charcoal) and to apply action research through experiments aimed at optimizing processes (with 82 tests before and after contractualization). The results have shown that parboiling rice after contracting gives a better husking yield and consequently less solid waste, ie a yield of $65.34 \%$ of rice and $34.66 \%$ of solid waste after contracting, against a yield of $56.93 \%$ of rice and $43.07 \%$ of waste before contracting. As for liquid waste, the average produced after contracting is 523.59 liters, lower than that before contracting which is 545.10 liters per $100 \mathrm{~kg}$ of steamed paddy, an increase of $4.10 \%$. With an annual processing capacity of 200 tonnes at CePEA, the quantity of solid waste produced is 69.3 tonnes (after contracting). The consumption of wood energy for the parboiling of $100 \mathrm{Kg}$ of paddy rice, is on average $18 \mathrm{Kg}$, or about 36 tonnes of wood annually. By using briquettes, the cost of fuel can be reduced by $30 \%$ to $40 \%$, saving time of 51.17 minutes for preheating and 61.93 minutes for steaming.
\end{abstract}

Keywords: Benin, wood energy, contracting, environment, rice parboiling

\section{Introduction}

Dans l'économie des pays ouest africains et dans la consommation des ménages urbains et ruraux, le riz joue un rôle important ${ }^{[1]}$. De nos jours, le riz est devenu une denrée de consommation courante et un produit stratégique pour une bonne partie de la population mondiale ${ }^{[2]}$. En guise d'exemple, la transformation du riz paddy en riz étuvé fait partie des activités post-récoltes qui consistent à séparer le riz en provenance du champ de son enveloppe protectrice après trempage et passage à la vapeur d'eau. Cette activité fait recours au chauffage à la vapeur d'eau en condition fermée en vue d'accroitre la valeur nutritionnelle et réduire le taux de brisure lors du décorticage. L'efficience de ces procédés dépend de l'optimisation du rendement de la transformation. En effet, la transformation du riz aboutit inévitablement à la production de coques de riz et de riz mal décortiqué, appelé communément balles de riz, et utilise de l'énergie et de l'eau ${ }^{[3]}$. L'optimisation vise à obtenir un meilleur rendement du riz commercialisable à la fin du cycle mais aussi à utiliser le minimum d'énergie et d'eau ${ }^{[2]}$, et par ricochet à gérer le minimum de déchets solides (balles de riz) et liquides (eaux usées). Selon le degré d'électro-mécanisation, on distingue grossièrement les transformations traditionnelle et moderne, et des transformations intermédiaires.

Au Bénin, les activités de transformation du riz étuvé sont l'apanage des femmes avec l'appui des enfants pour la collecte d'eau et le lavage du paddy ${ }^{[4]}$. Dans la commune de 
Natitingou, des groupements de femmes affiliées au Centre CePEA Sourou ${ }^{1}$ sont actives dans la transformation du riz étuvé de marque 'Sourou' à base d'une méthode semi traditionnelle où seul le décorticage est électro-mécanisé. Ces femmes utilisent le bois-énergie dans l'étuvage du riz. Créé en 2008 par SOS village d'Enfant Bénin et accompagné techniquement par GERME-ONG, ce centre vise à promouvoir l'autonomisation des femmes et la sécurité alimentaire à travers la transformation du riz. Le riz fait partie des filières prioritaires de développement Agricole au Bénin en conformité avec la stratégie Nationale de développement des filières agricoles ${ }^{[5]}$.

Après des difficultés de mobilisation de la matière première au démarrage, le centre avec l'aide de partenaires techniques et financiers a fini par recourir à la contractualisation avec les producteurs pour la fourniture de la matière première. Le volume de transformation s'est accru passant de 50 tonnes à 200 tonnes de riz paddy entre 2008 et 2014 par an ${ }^{2}$. Ce niveau de transformation est favorisé par la demande (consommation) de riz local qui augmente du fait des facteurs sociodémographiques et économiques des ménages [6]. Cependant, l'accroissement du volume de transformation entraîne une chaîne de contraintes liées aux besoins en eau, en bois-énergie et en temps ainsi qu'à l'augmentation des sous-produits et déchets de transformation. Ces contraintes posent des problèmes écologiques liés à la déforestation, des problèmes d'eau et des problèmes d'assainissement liés à l'amoncellement des balles de riz.

Pour atténuer ces contraintes liées à la déforestation, à l'eau et au temps, la technologie de foyers améliorés et de production de bio-charbon a été introduite. Une étude comparative a été faite avec ces trois déterminants de l'étuvage à partir de l'hypothèse selon laquelle l'adoption du foyer amélioré et du bio-charbon réduit la consommation en bois-énergie, en eau et en temps de cuisson dans un contexte d'accès au paddy de bonne qualité du fait de la contractualisation entre producteurs et transformatrices de riz. Dans ce contexte, si tel est que la contractualisation a permis d'améliorer le taux de rendement au décorticage du riz paddy, quels sont alors les effets environnementaux de l'étuvage du riz avant et après la contractualisation ?

Pour cela, le présent article analyse les effets environnementaux issus de l'étuvage du riz cinq après l'expérimentation de la contractualisation chez les transformatrices du riz paddy au Nord-Ouest du Bénin, en y rapportant les résultats obtenus à partir de différents tests.

\section{Cadre théorique}

Dans le cadre de cette recherche, deux principales théories ont été le fil conducteur: la théorie du développement durable et celle des contrats particulièrement celle de l'Agence.

Depuis 1987, le développement durable gagne progressivement du terrain dans une perspective d'institutionnalisation sans lien étroit avec les théories de développement ${ }^{[7]}$ et est, le plus souvent présent comme résultant d'un jeu d'interactions entre les acteurs avec des intérêts différents ${ }^{[8]}$. Dans une perspective de responsabilisation sociale et environnementale, les actions de développement économique doivent tenir compte des générations actuelles et futures. Dans le secteur agricole

\footnotetext{
${ }^{1}$ Centre de Promotion de l'Entreprenariat Agricole Féminin

${ }^{2}$ Statistiques annuelles de GERME-ONG (2017)
}

plusieurs pratiques dans la logique d'accroître le niveau de revenu, portent des préjudices à l'environnement ce qui implique un changement de pratiques. L'importance de ce changement n'est plus à démontrer dans les actions de développement dans la mesure où, de la mise en œuvre de ces pratiques apparaissent des enseignements techniques tout comme de : du faire au savoir ou l'inverse du savoir au faire en mettant les techniques en pratique ${ }^{[9]}$. Telle est la situation qui prévaut dans le cadre de l'étuvage du riz dont les pratiques ont des effets sur l'environnement. La résolution de ses problèmes donne naissance à de nouvelles techniques et pratiques (innovations). Dans le cadre de la présente étude la dimension développement durable sera analysée sur toutes les étapes de transformation du riz. C'est d'autant plus important quand on sait que la dimension écologique est plus souvent analysée au niveau du maillon production au niveau de plusieurs études ${ }^{[10]}$.

L'analyse des contraintes écologiques dans le processus d'étuvage est aussi en rapport avec la qualité du paddy qui, à des moments donnés, dépend de la nature des relations contractuelles. La contractualisation apparait donc comme une innovation qui contribue à réduire les risques environnementaux dans le processus d'étuvage du riz sur les déchets solides. La mise en relation entre les acteurs renvoie à la théorie de l'agence dans laquelle il y a un agent qui agit comme représentant du principal ${ }^{[11]}$. L'agence fait appel à un contrat qui engage plusieurs acteurs à travers les demandes et offres de services ${ }^{[12]}$. Dans le cas de la présente étude, les relations entre producteurs et transformatrices de riz étuvé afin d'accéder au paddy de qualité occupe une place prépondérante. Pour d'autres auteurs la contractualisation est une opportunité économique pour les acteurs de la filière riz ${ }^{[13]}$.

Par ailleurs, l'analyse de la dimension environnementale au niveau de la transformation, touche plus les entreprises d'autant plus que la transformation se fait au niveau des usines de transformation du riz. Dans la plupart des entreprises, la prise en compte des aspects écologiques peut être difficile dans l'application « puisque les responsabilités n'interagissent pas avec le choix des produits et marchés de l'entreprise dans le procédé de formulation de la stratégie, elles peuvent être traitées comme des contraintes. " [14]. Cette activité de transformation et ses impacts environnementaux sont tributaires de trois facteurs qui selon [15] sont: la population (P), le niveau de revenu et de consommation (Affluence A) et enfin la Technologie (T). Ces impacts (I) articulés aux causes ont donné mathématiquement l'expression conceptuelle (IPAT), qui fait objet de cette recherche.

\section{Matériel et méthodes}

\subsection{Présentation de la zone de recherche}

La présente recherche a été conduite au Nord-Ouest du Bénin, dans le département de l'Atacora, précisément dans la commune Natitingou. Située entre $10^{\circ} 19^{\prime}$ Nord et $1^{\circ} 23^{\prime}$ Est, la commune de Natitingou couvre une superficie de $3.045 \mathrm{~km}^{2}$ soit $12,8 \%$ de la superficie totale du département. Elle est limitée au Nord par la commune de Toucountouna, au Sud et à l'Est par celle de Kouandé et à l'Ouest par celle de Boukombé. La commune de Natitingou est caractérisée par la chaîne de l'Atacora d'où elle tient son relief accidenté, composé surtout de plateaux et de collines dont les vallées se présentent souvent en pente forte. 
Pour les activités de transformation, les contrats sont signés non seulement avec les producteurs de riz de la commune de Natitingou, mais d'autres producteurs des autres communes notamment Matéri, Cobly, Tanguiéta qui sont des fournisseurs de paddy.
Pour assurer l'étuvage du riz, les femmes étuveuses ont plusieurs sources d'approvisionnement situées dans les communes mitoyennes comme Boukombé, Toucountouna ou parfois Tanguiéta.

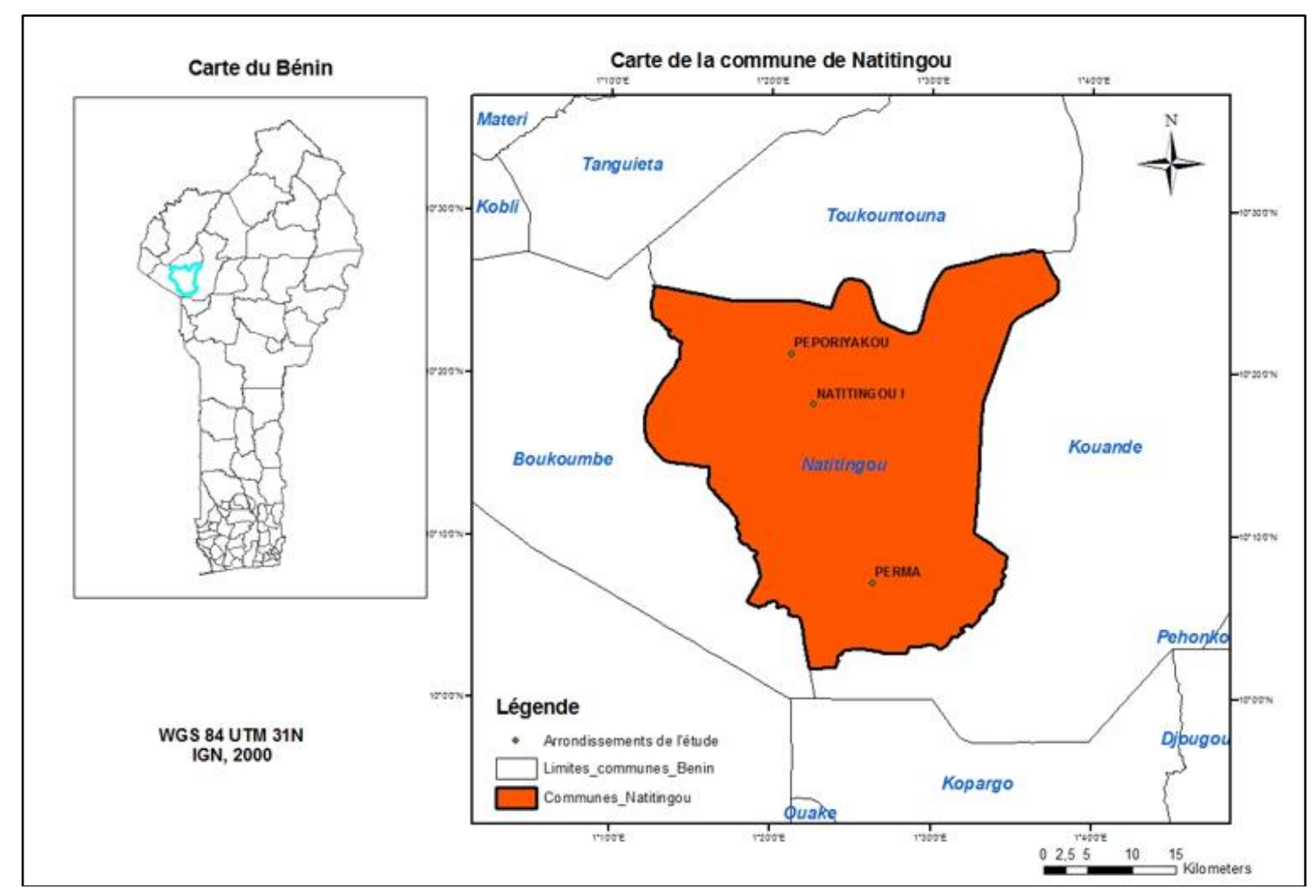

Fig 1: Présentation de la commune de Natitingou

\subsection{Echantillonnage et base de données}

L'unité de recherche représente les transformatrices du riz du CEPEA. La technique de choix raisonné a permis de catégoriser ces acteurs selon qu'ils aient participé au moins une fois à un mécanisme de contractualisation pendant les cinq dernières années avec pour année de référence 2012.

En premier lieu, la population mère est constituée de 124 transformatrices du riz paddy (dont 82 adoptants de la contractualisation) des communes de Natitingou, de Cobly et de Djougou. Pour des raisons de suivi rapproché dans l'expérimentation, les femmes transformatrices de riz du CePEA Sourou de la commune de Natitingou, ont fait objet d'enquête et d'expérimentation.

Ainsi, après un diagnostic participatif, deux phases des expérimentations se sont déroulées par la constitution de groupes de femmes avec qui les tests ont été faits.

Pour cela, vingt-quatre ${ }^{[24]}$ femmes transformatrices ont été tirées au hasard à raison de trois au niveau de chacun des huit (08) groupements que compte le centre CePEA Sourou. A ces femmes étuveuses s'ajoutent l'équipe d'encadrement du centre composé des agents de GERME-ONG. Quatre agents d'appui technique ont été choisis et sont répartis au hasard au niveau des quatre groupes de femmes étuveuses afin de faciliter l'enregistrement des données au niveau de fiches de collecte de données.

En effet, chaque groupe de femmes transforme de façon consécutive en moyenne 10 sacs de $100 \mathrm{~kg}$ de paddy tiré au hasard du magasin de stockage du centre CePEA Sourou. Une fois les sacs tirés, il est procédé à la mesure des poids, et des ajustements sont faits afin que tous les sacs aient le même poids $(100 \mathrm{~kg})$.
Au total, 41 répétitions ont été faites en 2012 puis 82 en 2017 à raison de 41 tests avec bois-énergie comme combustibles et 41 tests avec les briquettes de bio charbon comme combustible. Ces deux phases sont totalement différentes du point de vue qualité du paddy.

\subsection{Conditions de déroulement et phases des expérimentations}

La recherche s'est faite en milieu réel au niveau du centre de transformation du riz Sourou de l'association des femmes étuveuses Sourou Bayayé à Natitingou. En raison de la spécificité de l'étuvage, il nous est apparu nécessaire d'associer les acteurs du centre (les transformatrices de riz, l'équipe d'encadrement du centre et l'équipe de GERME $\mathrm{ONG}$ ) à chaque étape de la recherche qui s'inscrit dans une démarche de recherche-action.

Pour éviter de perturber les activités de transformation des femmes, les expérimentations ont lieu une fois par semaine de juillet à décembre soit trois mois en saison des pluies et trois mois en saison sèche.

Deux principales phases ont caractérisé cette étude :

\section{Première phase}

La période de juillet à décembre 2012 qui correspond à la première phase, au cours de laquelle, les femmes étuveuses du CePEA Sourou, ne signaient pas de contrat avec les producteurs afin d'exiger des normes de qualité du paddy. Cette première phase a démarré par un diagnostic participatif qui a permis de ressortir les différentes contraintes écologiques de l'étuvage du riz et de tracer les pistes de solution en termes de mesures d'atténuation. 
Dans la perspective de ressortir toutes les contraintes écologiques et solutions éventuelles, ce diagnostic participatif s'est fait à travers des groupes de discussion et focus groups avec les femmes transformatrices de riz, l'équipe d'encadrement, GERME-ONG, l'Agence Territoriale pour le Développement Agricole (déconcentration du ministère de l'agriculture au niveau communal). La production de briquettes de bio charbon et la contractualisation entre producteurs et transformatrices ont été des solutions retenues et qui doivent faire objet de recherche-action.

Pour mieux apprécier ces contraintes écologiques, 41 tests d'étuvages ont été réalisés. Cette première phase a permis de définir ensemble avec les acteurs du centre et l'équipe d'encadrement et l'équipe de GERME ONG du choix d'un dispositif de recherche ainsi que les modalités de sa conduite.

\section{Deuxième phase}

La seconde phase de juillet à décembre 2017, période correspondant à la cinquième année de contractualisation entre producteurs et les femmes étuveuses de riz du CePEA Sourou ainsi que la recherche-action sur la transformation des balles de riz en briquettes de bio-charbon. Cette phase a démarré avec la restitution des résultats de première phase et l'implication d'autres acteurs comme le Centre Régional de Recherche Agronomique, la Direction Départementale de l'environnement, les Partenaires Techniques et Financiers (ProAGRI, ENABEL, Louvain Développement). Il fallait donc refaire les expérimentations à travers la même démarche qu'en 2012. Cette seconde phase a été décomposée en deux sous-composantes : la première composante est relative à la reprise des expériences de 2012, cinq ans après la contractualisation entre producteurs et transformatrices. La seconde composante a permis de réaliser 41 autres tests afin de comparer le temps d'étuvage et le coût de combustible entre l'énergie bois et l'énergie bio charbon dans le processus d'étuvage.

$\mathrm{Au}$ cours des deux phases d'expérimentation, les kits d'étuvage utilisés sont identiques en dehors des foyers et combustibles. Les combustibles utilisés lors de l'expérience de 2012 sont uniquement du bois-énergie. En 2017, les deux types d'expérimentation ont été effectuées en utilisant le bois comme combustible ainsi que les briquettes de biocharbon.

\subsection{Collecte des données}

La collecte des données s'est faite à travers trois principaux outils:

- La présente étude s'est faite à travers un diagnostic participatif dans la perspective de mieux décrire la situation que vivent les acteurs et d'avoir la perception du problème par les acteurs. Pour faciliter la collecte des données, un guide d'entretien a été utilisé ainsi que le journal de bord qui permet de consigner les données sur les différents événements lors de l'étude. Ce diagnostic a été fait à travers des groupes de discussion et des focus group, un guide d'entretien a permis de collecter les données qualitatives. Pour une bonne relation de cause à effet entre contraintes écologiques, l'outil "'arbre à problème"' a été utilisé.

- Pour la collecte des données quantitatives, une fiche d'enregistrement des données a servi de questionnaire et a été utilisé pour collecter à chaque test les données sur le niveau de production des différents types de déchets.

- Les différentes solutions trouvées sont testées avec l'ensemble des acteurs engagés et des informations sont collectées à travers un guide d'entretien et un questionnaire.

\section{Les différentes données collectées sont}

- La quantité de déchets liquides: la quantité d'eau utilisée à chaque étape de l'étuvage. L'instrument de mesure est un fût de $200 \mathrm{~L}$ gradué qui est rempli au début de l'activité et à la fin de chaque opération le reste est mesuré. Cette différence entre la quantité initiale et celle finale permet d'évaluer la quantité d'eau utilisée.

- La quantité de bois énergie ou bio-charbon: Le combustible est pesé avec une balance électronique de portée $150 \mathrm{~kg}$ avec affichage numérique avant le démarrage de chaque opération et les pesées sont faites après l'opération. La différence est la masse de bois utilisée.

- La quantité de son et de balles de riz produite après décorticage est pesée à l'aide d'une balance après avoir recueilli le son. L'avantage est que l'usine de décorticage de riz du centre CePEA sépare le son et les balles.

- Le temps d'étuvage selon le type de combustible a été mesuré par un chronomètre.

\subsection{Analyse de données}

Une fois les expérimentations réalisées, l'analyse des données a été faite dans un premier temps à travers la détermination des paramètres de position et de dispersion des différentes variables qui seront exprimés par des tableaux et des graphiques.

Dans un deuxième temps, des comparaisons de moyennes ont été effectuées grâce au logiciel SPSS.21. Il s'agit surtout de la comparaison du niveau de production des déchets avant et après la contractualisation en tenant compte de la qualité du paddy. D'autre part, il est question de comparer les moyennes relatives aux coûts des combustibles et au temps d'étuvage déchets avant et après la contractualisation. Principalement il s'agit de faire la comparaison de deux (02) moyennes. Le test $\mathrm{T}$ de Student a donc été effectué pour la comparaison des moyennes issues des deux expérimentations.

On a:

H0: Pdéch2017 - Pdéch2012=0 contre H1: Pdéch2017 Pdéch2012 $\neq 0$

Avec Pdéch2012 et Pdéch2017 les niveaux de productions moyennes de déchets en 2012 (avant la contractualisation) et en 2017 (après la contractualisation).

Le logiciel a donné la valeur t de Student, le degré de liberté et la probabilité de signification p. Si le p donné est inférieur au seuil critique de $\alpha=5 \%$, alors on rejettera H0 et on acceptera H1. De plus, si Pdéch2017 < Pdéch2017, on conclut que le niveau de production de déchets après contrat en 2017 est inférieur à celui avant contrat en 2012, c'est-àdire que l'hypothèse de recherche est vérifiée. Dans le cas contraire, elle ne l'est pas.

\section{Résultats}

4.1 Caractéristiques des femmes étuveuses et gestion des déchets au centre CePEA 
Dans la zone de recherche, la transformation du paddy en riz étuvé est la principale activité exercée par les femmes enquêtées. Notons que ces transformatrices sont âgés en moyenne de 45,89 $( \pm 11,42)$ ans. Elles ont moyennement capitalisé $12,47( \pm 4,69)$ années d'expériences dans la transformation du riz paddy avec au moins quatre (04) ans dans la contractualisation. Ces femmes vivent dans des ménages. Néanmoins, cette activité de transformation à laquelle s'adonne ces femmes n'est pas sans conséquences sur l'environnement.

A la suite du diagnostic participatif et des discours des acteurs, il ressort trois principaux problèmes environnementaux à chaque étape du processus d'étuvage : la production de déchets solides, la production de déchets liquides et la déforestation. Le lavage du paddy nécessite l'utilisation de l'eau et qui par la suite est déversée dans la nature. Le trempage et l'étuvage quant eux, dépendent du bois-énergie. Une fois le processus d'étuvage achevé, le décorticage engendre une production de déchets solides (balles et son de riz).

\subsection{Production des déchets solides et liquides au cours du processus d'étuvage}

Dans la perspective d'impliquer les différents acteurs dans la résolution du problème lié aux déchets issus de l'étuvage $\mathrm{du}$ riz, et faciliter l'adoption des innovations qui en découleraient, des tests ont été réalisés avec l'ensemble des acteurs clés.

Table 1: Evaluation du niveau de production de déchets et du rendement au décorticage du riz

\begin{tabular}{|c|c|c|c|c|c|c|}
\hline Paramètres & Type & $\mathbf{N}$ & Moyenne & Ecart-type & Erreur standard & Test $\mathrm{t}$ de Student \\
\hline \multirow{2}{*}{ Rendement au décorticage du riz (\%) } & 2017 & 41 & 65,341 & 1,37 & 0,214 & \multirow{2}{*}{$\mathrm{t}=11,068 ; \mathrm{ddl}=80 ; \mathrm{p}=0,000$} \\
\hline & 2012 & 41 & 56,926 & 4,67 & 0,729 & \\
\hline \multirow{2}{*}{ Poids des déchets solides $(\mathrm{kg})$} & 2017 & 41 & 34,66 & 1,28 & 0,200 & \multirow{2}{*}{$\mathrm{t}=13,455 ; \mathrm{ddl}=80 ; \mathrm{p}=0,000$} \\
\hline & 2012 & 41 & 43,07 & 4,83 & 0,755 & \\
\hline \multirow{2}{*}{ Quantité de déchets liquides (L) } & 2017 & 41 & 225,171 & 5,58 & 0,872 & \multirow{2}{*}{$\mathrm{t}=16,109 ; \mathrm{ddl}=80 ; \mathrm{p}=0,000$} \\
\hline & 2012 & 41 & 256,317 & 11,04 & 1,725 & \\
\hline \multirow{2}{*}{ Quantité totale d'eau (L) } & 2017 & 41 & 523,59 & 11,95 & 1,866 & \multirow{2}{*}{$\mathrm{t}=-7,160 ; \mathrm{ddl}=80 ; \mathrm{p}=0,000$} \\
\hline & 2012 & 41 & 545,10 & 15,07 & 2,355 & \\
\hline
\end{tabular}

Source : Résultats d'expériences, 2012 et 2017.

$2017=$ Contexte de contractualisation avec les producteurs

$2012=$ Contexte d'absence de contrats avec les producteurs

Il ressort des résultats issus de cette recherche que le processus d'étuvage du riz a généré $34,66 \%$ de déchets solides en 2012 en absence de contrats avec les producteurs contre $43,07 \%$ dans un contexte de contractualisation avec les producteurs en 2017. En effet, les quantités de déchets solides (balles de riz et autres) du riz étuvé dans un contexte de contractualisation sont donc inférieures aux moyennes obtenues en 2012 (sans contractualisation) avec une différence significative au seuil de $1 \%(P<0,05)$.

En termes de déchet liquide également, la quantité moyenne d'eau utilisée pour le lavage du riz dans un contexte de contractualisation $(225,171 \mathrm{~L})$ avec les producteurs est inférieur à celle obtenue hors contrat $(256,317$ L) en 2012 avec une différence significative $(P<0,05)$.

De même, la quantité moyenne de la totalité d'eau utilisée pour l'étuvage du riz dans un contexte de contractualisation $(523,59 \mathrm{~L})$ avec les producteurs est inférieur à la moyenne obtenue en 2012 (sans contractualisation, 545,10 L). Mais la différence de moyenne obtenue n'est pas significative $(P>0,05)$.

Cependant, il est constaté que le rendement au décorticage du riz étuvé dans un contexte de contractualisation avec les producteurs est supérieur au rendement obtenu en 2012 (sans contractualisation) avec une différence de $8,41 \mathrm{~kg}$ de riz usiné sur $100 \mathrm{~kg}$ de paddy transformé. Cette différence est significative au seuil de 5\%. De ce fait, la contractualisation a également eu d'incidence sur le niveau de production des déchets solides et liquides du fait de la qualité du riz paddy.

\subsection{Temps mis à l'étuvage selon le type de combustible}

L'analyse des résultats du tableau 2 révèle qu'avec le combustible «briquette de bio-charbon » fabriqué à base de balle de riz, les femmes transformatrices réalisaient un gain de temps de 51,17 min (plus ou moins 1,13 min) en comparaison avec le temps d'utilisation du combustible «bois» pour le préchauffage. La différence de moyennes obtenue à partir des résultats est significative au seuil de $5 \%$ $(p<0,05)$. Aussi, avec le combustible «bois-énergie», la moyenne de temps consacré à l'étuvage est supérieure au temps du combustible « briquette de bio-charbon ».

Sur la base du coût des combustibles, les moyennes obtenues prouvent que le «combustible bois-énergie» (1000,05 FCFA) revenait plus cher que les briquettes de biocharbon $(617,68$ FCFA) avec une marge différentielle de 382,24 FCFA (+/- 27,46 FCFA). Cette différence de moyenne est significative au seuil de de $5 \%(p<0,05)$. Cette différence prouve que les briquettes sont économiquement plus efficaces en termes de réduction de coût pour un même niveau de rendement.

Table 2: Comparaison du temps lors de l'étuvage selon le type de combustible et coût du combustible

\begin{tabular}{|c|c|c|c|c|c|c|}
\hline Paramètres & Type de combustible & $\mathbf{N}$ & Moyenne & Ecart-type & Erreur standard & Test t de Student \\
\hline \multirow{2}{*}{ Temps de préchauffage (minute) } & Bio-charbon & 41 & 68,293 & 2,60 & 0,406 & $\mathrm{t}=16,228 ; \mathrm{ddl}=80 ;$ \\
& Bois-énergie & 41 & 119,463 & 6,76 & 1,057 & $\mathrm{p}=0,000$ \\
\hline \multirow{2}{*}{ Temps d'étuvage (minute) } & Bio-charbon & 41 & 46,049 & 3,28 & 0,513 & $\mathrm{t}=13,824 ; \mathrm{ddl}=80 ;$ \\
\cline { 2 - 7 } & Bois-énergie & 41 & 107,976 & 8,09 & 1,264 & $\mathrm{p}=0,000$ \\
\hline \multirow{2}{*}{ Cout du combustible (F CFA) } & Bio-charbon & 41 & 617,68 & 39,14 & 6,114 & $\mathrm{t}=33,460 ; \mathrm{ddl}=80 ;$ \\
\cline { 2 - 6 } & Bois-énergie & 41 & 1000,05 & 171,45 & 26,777 & $\mathrm{p}=0,000$ \\
\hline
\end{tabular}

Source : Résultats d'expériences de 2012 et 2017

4.4 Niveau de consommation de l'énergie-bois 
Les différentes expériences réalisées en 2012 et en 2017 ont permis de ressortir le niveau de consommation du bois durant le processus d'étuvage avant et 5 ans après le processus de contractualisation.
En effet, l'étuvage de $100 \mathrm{~kg}$ de riz paddy nécessitait environ 18,18 et $19,44 \mathrm{~kg}$ de bois quel que soit la nature des relations entre producteurs et transformatrices. Mais cette différence n'est pas significative $(p>0,05)$.

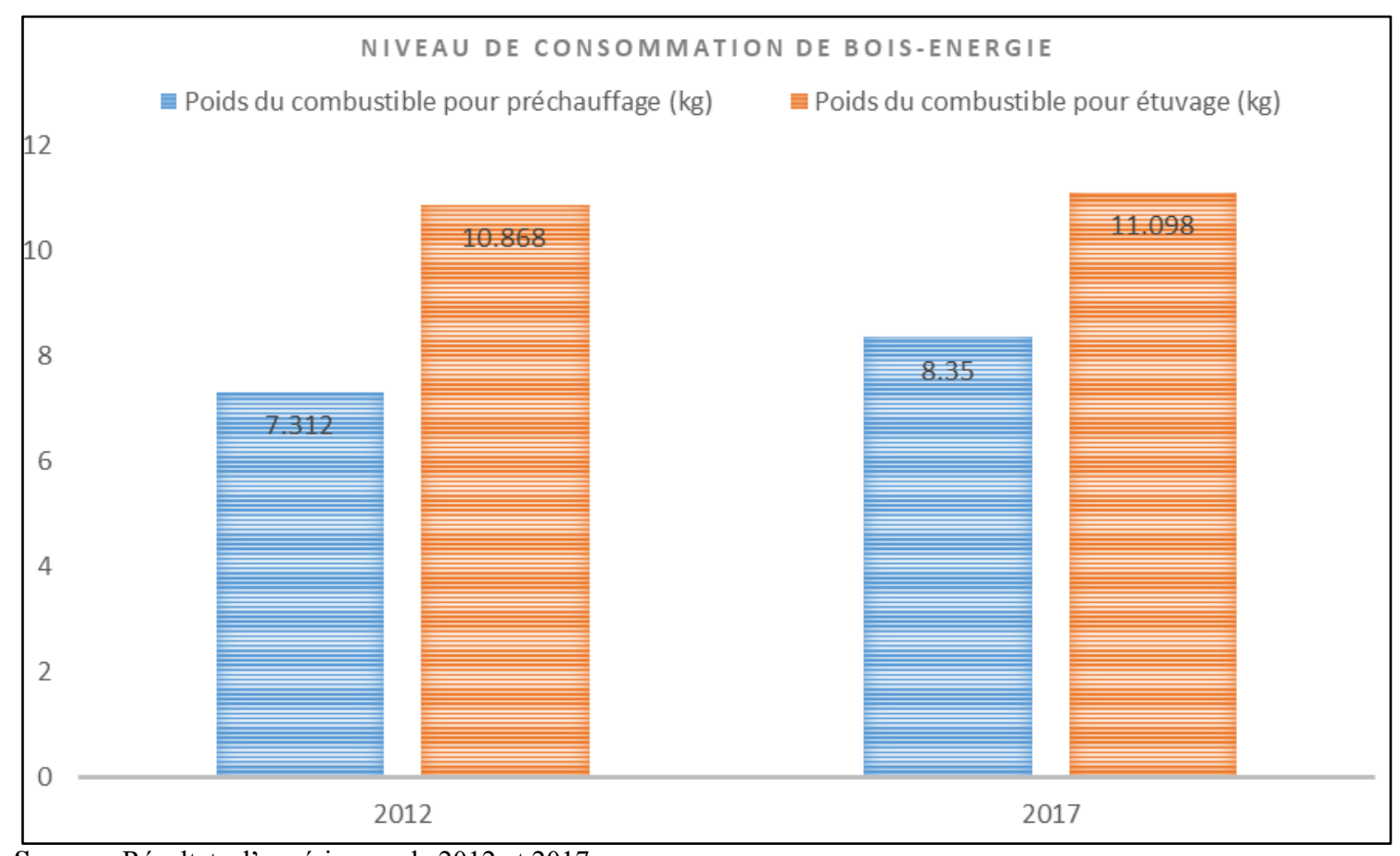

Source : Résultats d'expériences de 2012 et 2017

Fig 2: Consommation de bois dans le processus d'étuvage du riz

\section{Discussion}

Les résultats de cette recherche ont montré que la contractualisation entre les transformatrices et les producteurs du riz a amélioré le rendement du riz étuvé dans la période. De ce fait, cet effet positif de la contractualisation sur le rendement au décorticage du riz étuvé a également réduit les quantités de déchets solides (immatures, balles et son) du riz étuvé. Ainsi, cette variation du niveau de production de déchet dépend de la qualité du riz paddy qui est fonction de la nature des relations d'affaires entre producteurs et transformatrices. En effet, les exigences contractuelles permettent aux transformatrices d'accéder à une bonne qualité de riz paddy avec un taux d'impureté relativement bas. Le niveau d'impureté agit sur le rendement après décorticage et par ricochet sur les marges bénéficiaires. En outre, les balles de riz qui sont de l'ordre de 20 à $25 \%$ du poids du riz paddy transformé causent des problèmes d'assainissement au niveau des unités de transformation de riz. Ce résultat rejoint les conclusions de ${ }^{[3]}$ qui ont montré que les différentes étapes de la transformation du paddy libèrent une énorme quantité de déchets solides, principalement des balles de riz, des cendres de balles de riz et des déchets liquides sous forme d'eaux usées de l'industrie du riz en Inde.

Ces déchets viennent s'ajouter aux déchets ménagers qui constituent déjà de grands problèmes de développement dans la mesure où, au Bénin, les déchets ménagers sont collectés sans un véritable traitement ${ }^{[16]}$.

De plus, les déchets solides sont produits aussi bien dans le cadre du riz blanc que du riz étuvé. Cependant, la principale particularité lors de l'étuvage du riz est la production des déchets liquides car l'étuvage du riz est tributaire de l'eau. En effet, l'étuvage de100 $\mathrm{kg}$ de paddy nécessitait respectivement $523,59 \mathrm{~L}$ et $545,10 \mathrm{~L}$ d'eau en moyenne pour les expérimentations en 2017 et 2012. Une extrapolation sur la base de ce besoin en eau après les tests ressort qu'il faut en moyenne un volume d'eau équivalent à $1047.18 \mathrm{~m}^{3}$ d'eau pour transformer 200 tonnes de paddy dans un contexte de contractualisation. Cette consommation d'eau dans le cadre de l'étuvage vient s'ajouter aux besoins d'eau de consommation [17]. On estime, qu'avec une population de 13243000 habitants en 2025, la quantité d'eau disponible par habitant et par an serait de $990 \mathrm{~m} 3$ (Ministère de l'Energie et de l'Eau, 2008). En l'absence donc d'un mécanisme de gestion des eaux après l'étuvage, toute la quantité d'eau utilisée est déversée dans la nature et constituent ainsi une source de pollution s'ajoutant aux déchets solides.

Mais les problèmes environnementaux dans le cadre de l'étuvage ne se limitent pas à la pollution mais est également source de déforestation du fait de l'utilisation de bois comme combustible.

En ce qui concerne l'utilisation de combustible lors de l'étuvage, dans les analyses des pratiques favorisant la perte des ligneux, la production agricole est souvent citée et principalement l'igname ${ }^{[19]}$. Rarement des allusions sont faites au niveau de la production agricole. De nos jours, de nombreuses études s'intéressent plus à la dimension accroissement de la valeur ajoutée de la transformation ${ }^{[13]}$. Dans le cadre de l'étuvage, les principales étapes sont très dépendantes de l'utilisation de bois ou charbon de bois entrainant une pression sur les forêts.

En effet, le niveau d'utilisation de bois suppose qu'annuellement, les femmes étuveuses utilisent environ 36 tonnes de bois pour pouvoir mener leurs activités. Dans cette dynamique, il faut ajouter que le couvert végétal se dégrade et d'après les études de la FAO, environ 70.000 ha de forêt sont défrichés au cours de la décennie 1990 et 2000 
du fait du développement de l'agriculture, de l'élevage, etc. Pour ce qui concerne la masse des bois pour le chauffage, il existe très peu de données dans plusieurs pays africains [20]. Ces différentes préoccupations environnementales donne une place de choix au développement durable à travers trois (03) sphères qui sont étroitement imbriquées : protection de l'environnement, gain économique et le progrès social ${ }^{[21]}$.

Selon le rapport du PNUD $^{3}$, la consommation de biomasse en 2010 au Bénin a estimé la consommation nationale à 4 297522 tonnes de bois et 245197 tonnes de charbon de bois. Le développement des activités de transformation agroalimentaire qui est encore à l'étape semi-artisanale a une incidence sur l'environnement. Pour atténuer ces effets, le centre Sourou Bayayé a développé plusieurs mécanismes. « Le comportement en combustion des produits obtenus est proche de celui du charbon de bois mais avec un rendement nettement supérieur (comparaison avec l'expérience malgache au charbon avec boulet en fourneau BIOTERRE) » ${ }^{[22]}$. Cet avantage a été également ressorti à travers les travaux de ${ }^{[23]}$.

Enfin, les effets pervers de l'étuvage sur l'environnement peuvent être atténués à travers plusieurs stratégies. Parmi les plus appliquées, on peut citer : le bio-char, les briquettes et les eaux usées. Par exemple, la production de bio-char à partir des balles de riz se fait à travers un dispositif de pyrolyse. Ce bio-char est utilisé pour la production maraichère dans une perspective d'améliorer la texture du sol ${ }^{[24]}$. Aussi, la production des briquettes à partir des balles de riz peut être faite par une mini-unité de production de briquette qui est installée en vue de réduire l'utilisation du bois au niveau du site. Et, le recyclage des eaux usées issues de lavage du riz pour la production maraîchère et en biogaz [25]. Mais les femmes ont ressorti que les eaux issues de l'étuvage et du trempage ne sont pas encore utilisées et l'une des femmes a fait cette déclaration : « quand nous utilisons de l'étuvage où du trempage nos plants meurent $»$. La recherche documentaire à ce sujet ressort la présence de silice au niveau des balles de riz. La balle de riz est très riche en cellulose brute $(40 \%)$ et en silice $(20 \%)^{[22]}$.

\section{Conclusion}

$\mathrm{Au}$ regard de tout ce qui précède, il ressort de cette recherche que l'étuvage du riz a un effet négatif sur l'environnement à travers la pression sur les forêts du fait de l'utilisation de plus en plus croissante de bois de chauffe d'une part et la production de déchets liquides et solides d'autre part. Cette production de déchets solides (balles de riz) en tant que biomasse est recyclable et constitue une opportunité économique. Cette biomasse peut être transformée en briquette constituant ainsi une source alternative de combustible. L'utilisation de ce type de combustible règle simultanément le problème d'encombrement des balles de riz et de forte dépendance au bois-énergie lors de l'étuvage du riz avec un gain de temps. Aussi, cette biomasse est une matière première pour la production du bio-char dont les recherches scientifiques ressortent de plus en plus les effets agronomiques. En ce qui concerne les déchets liquides qui sont de deux ordres dont l'un est issu du trempage et de l'étuvage puis l'autre du lavage lors du processus d'étuvage. Il faut noter que les déchets liquides issus du lavage sont recyclés par les

${ }^{3}$ PNUD. 2016. Renforcement de la résilience du secteur de

l'énergie aux impacts des changements climatiques au Bénin. femmes du CePEA Sourou pour le maraichage autour de leur unité d'étuvage et le surplus d'eau est utilisé pour l'extinction du feu lors de la production du bio-char.

\section{Remerciements}

Les auteurs de l'article remercient sincèrement l'Unité Fonctionnelle Régionale Atacora-Donga de ENABEL Bénin qui a permis la réalisation de ces expériences au niveau du centre de transformation des femmes de Sourou Bayayé. Wilma BASS, G. VAN Nieuwenhuysen et Amadou DIENG je vous prie de recevoir un des fruits de vos engagements pour l'impulsion des dynamiques d'innovations. Nos remerciements vont également à l'endroit de toute l'équipe de GERME-ONG qui a assuré techniquement le suivi des opérations. Nous ne saurons finir les remerciements sans citer Louvain Coopération au Développement qui à travers ' 'ingénieux sud', a envoyé des stagiaires qui ont beaucoup contribué à la réussite des expérimentations. Aux femmes étuveuses de «Sourou Bayayé » recevez à travers cet article l'expression de notre reconnaissance pour vos efforts méritoires.

\section{Références}

1. Fall AA. Chaine de valeur riz en Afrique de l'Ouest: Performance, enjeux et défis en Côte d'Ivoire, Guinée, Libéria, Mali, Sierra Léone et Sénégal. J Appl Biosci. 2018; 130:13175-13186.

2. Ahmadi N, Bouman B. Riz et rizicultures, enjeux économiques, écologiques et scientifiques. Cah Agric. 2013; 22(5):333-335.

3. Kumar A, Roy A, Priyadarshinee R, Sengupta B, Malaviya A, Dasguptamandal D et al. Economic and sustainable management of wastes from rice industry: combating the potential threats. Environ Sci Pollut Res. 2017; 24(34):26279-26296.

4. Hinnou LC, Mongbo RL, Agboh-Noameshie AR. Equité-genre et autonomisation des femmes dans la chaîne de valeur du riz local étuvé au Bénin. 10esJournées Rech En Sci Soc. 2015, 30.

5. MAEP. Plan Stratégique de Relance du Secteur Agricole (PSRSA). MAEP, 2011.

6. Kinkpe TA, Adegbola PY, Yabi JA, Adekamni S, Biaou G. Déterminants de la consommation du riz local en Afrique de l'ouest: cas du Benin. In Addis Abeba, Ethiopie, 2016.

7. Gendron C, Révéret JP. Le développement Durable. Econ Société. Sept. 2000; (37):111-24.

8. Lauriol J. LE Développement Durable À LA Recherche D'UN Corps DE Doctrine. Rev Fr Gest. 2004; 5(152):137-50.

9. Landais E, Deffontaines JP, Benoît M. Les pratiques des agriculteurs. Point de vue sur un courant nouveau de la recherche agronomique. Persée. 1988; (109):125-58.

10. Adégbola PY, Akoha S, Diallo B. Analyse de la compétitivité du riz local au Bénin. Programme Renf Rech Sur Sécurité Aliment En Afr L’Ouest Mich State Univ Mich E-U, 2011,

11. Ross M. The economic theory of agency: the principal problem. Am Econ Rev, 1973.

12. Jensen M, Meckling W. Theory of the firm: managerial behavior, agency costs and capital structure. J Financ Econ, 1976. 
13. Soulé BG. La contractualisation: une opportunité pour la promotion des chaines de valeur du riz local en Afrique de l'Ouest. East Lansing, MI: Michigan State University, SRAI; 2016, 34.

14. Ansoff I. Corporate strategy. Mc Graw-Hill. Bungay, Suffolk. 1968, 59.

15. Ehrlich PR. 77ie Population Bomb. New York: Ballantine Books, 1968.

16. Gbinlo R. Organisation et financement de la gestion des déchets ménagers dans les villes de l'Afrique Subsaharienne : le cas de la ville de Cotonou au Bénin. Universite D'orleans, 2010.

17. Mikunthan T, Balakumar S, Prabhaharan M. Management of paddy soaking water: as a source for enriched compost making, 2012;

18. Ministre de l'Energie et de l'Eau. Politique Nationale DE L'EAU. Ministère de l'Eau et de l'Energie, 2008.

19. Gibigaye M. Effets environnementaux de la production de l'igname sur le système agroforestier dans la commune de Ouaké au Bénin. Int J Biol Chem Sci. 2013; 7(3):961-977.

20. Bertrand A. Les problèmes du bois de chauffage et du charbon de bois en Afrique tropicale. Juin. 1977; (173):39-48.

21. Peracod/Gtz. Mise en oeuvre d'unité pilote de production de biocharbon du typha et mesure de rentabilité économique de l'activité. Sénégal: GTZ, 2008.

22. GTZ. Le biocharbon: quelles opportunités pour le Sénégal?, 2009; (11).

23. Koala L. Fabrication manuelle de briquettes de balles de riz et évaluation des performances du foyer amélioré à balles de riz. [Burkina Faso]: Université Polytechnique de Bobo Dioulasso, 2012.

24. Barry H, Major J. le biochar comme amendement du sol au Québec: résultats agronomiques de quatre ans d'essais terrain, 2011.

25. Nadaleti WC. Utilization of residues from rice parboiling industries in southern Brazil for biogas and hydrogen-syngas generation: Heat, electricity and energy planning. Renew Energy. 2019; 131:55-72. 